\title{
Numerical analysis of MHD stagnation point flow towards a radially stretching convectively heated disk
}

\author{
Stanford Shateyi \\ University of Venda, \\ South Africa
}

\author{
Daniel Makinde \\ Cape Peninsula University of Technology, \\ South Africa
}

\begin{abstract}
The steady stagnation point flow and heat transfer of an electrically conducting incompressible viscous fluid is extended to the case where the disk surface is convectively heated and radially stretching. The fluid is subjected to an external uniform magnetic field perpendicular to the plane of the disk. The governing momentum and energy balance equations give rise to non-linear boundary value problem. Using a spectral relaxation method with a Chebyshev spectral collocation method, the numerical solutions are obtained over the entire range of the physical parameters. Emphasis has been laid to study the effects of viscous dissipation and Joule heating on the thermal boundary layer. Pertinent results on the effects of various thermophysical parameters on the velocity and temperature fields as well as local skin friction and local Nusselt number are discussed in detail and shown graphically and/or in tabular form.
\end{abstract}

Keywords-Radially stretching disk; Stagnation point flow; Magnetic field; Convective heating.

\section{INTRODUCTION}

Stagnation-point flow appears in virtually all fields of science and engineering. A flow can be stagnated by a solid wall or a free stagnation-point or a line can exist in the interior of the fluid domain. The study of stagnationpoint flow was pioneered by Hiemenz in 1911, who solved the two dimensional stagnation-point problem using a similarity transformation. Motsa et al. (2012) formulated the Maxwell fluid for two-dimensional stagnation flow towards a shrinking sheet and then calculated the numerical solution of transformed nonlinear ordinary differential equations via the successive linearisation method. Bhattacharyya et al. (2011) analyzed the effects of partial slip on steady boundary layer stagnationpoint flow of an incompressible fluid and heat transfer

S. Shateyi is with the Department of Mathematics and Applied Mathematics, University of Venda, , X5050, 0950 South Africa e-mail: stanford.shateyi@univen.ac.za.

O.D. Makinde is with the Institute for Advanced Research in Mathematical Modeling and Computations,Cape Peninsula University of Technology, P. O. Box 1906, Bellville 7535, South Africa. Email: makinded@cput.ac.za

Manuscript received July, 2013 towards a shrinking sheet. This investigation explored the conditions of the non-existence, existence, uniqueness and duality of the solutions of self-similar equations numerically. Makinde and Charles (2012) presented computational dynamics of hydromagnetic stagnation flow towards a stretching sheet.

Governing equations modelling MHD flow and heat transfer over stretching surfaces are highly nonlinear. To that end, exact solutions are impossible to obtain. Therefore numerical solutions have always been developed, modified and highbred, as a bid of getting more accurate and stable solutions. The current study seeks to extend the steady stagnation point flow and heat transfer of an electrically conducting incompressible viscous fluid to the case where the disk surface is convectively heated and radially stretching. We propose to numerically solve the present problem using a recently developed iterative method known as spectral relaxation method (SRM) see Motsa et al. (2012). The SRM approach is based on transforming nonlinear ordinary differential equation into an iterative scheme. The iterative scheme is then blended with chebyshev spectral method.

\section{MOdEL Formulation}

We consider a steady stagnation-point flow of an electrically conducting incompressible viscous fluid towards a radially stretching convectively heated disk in the presence of a transverse magnetic field of strength $B_{0}$ applied parallel to the z-axis. The sheet is stretched with a linear velocity $u=U_{w}(r)=a r$, where $a$ is a real number. The bottom surface of the plate is assumed to be heated by convection from a hot fluid at temperature $T$, which provides a heat transfer coefficient, $h$. The induced magnetic field due to the motion of the electrically conducting fluid is negligible. It is also assumed that the external electrical field is zero and that the electric field due to the polarization of charges is negligible. The relevant governing equations of fluid flow and heat transfer are, 


$$
\begin{aligned}
& \frac{\partial}{\partial r}(r u)+\frac{\partial}{\partial z}(r w)=0 \\
& u \frac{\partial u}{\partial r}+w \frac{\partial u}{\partial z}=U_{\infty} \frac{d U_{\infty}}{d r}-\frac{\sigma B_{0}^{2}\left(u-U_{\infty}\right)}{\rho} \\
& +\nu \frac{\partial^{2} u}{\partial z^{2}} \\
& u \frac{\partial T}{\partial r}+w \frac{\partial T}{\partial z}=\frac{k}{\rho c_{p}} \frac{\partial^{2} T}{\partial z^{2}} \\
& +\frac{\mu}{\rho c_{p}}\left(\frac{\partial u}{\partial z}\right)^{2}+\frac{\sigma B_{0}^{2}\left(u-U_{\infty}\right)^{2}}{\rho}
\end{aligned}
$$

with

$$
\begin{aligned}
u=U_{w}=a r, w & =0,-k \frac{\partial T}{\partial z}=h\left(T_{f}-T\right) \text { at } z=0, \\
u & \rightarrow U_{\infty}=b r, T \rightarrow T_{\infty} \text { as } z \rightarrow \infty .
\end{aligned}
$$

\section{A. Similarity Transformation}

To make the problem amenable, following Butt and Ali (2013), among others, we introduce the following nondimensional quantities:

$$
\begin{aligned}
& \eta=\sqrt{\frac{b}{\nu}} z, u(r, z)=b r f^{\prime}(\eta), w(r, z)=-2 \sqrt{b \nu} f(\eta) \\
& \theta(\eta)=\frac{T-T_{\infty}}{T_{w}-T_{\infty}}, \nu=\frac{\mu}{\rho}, \lambda=\frac{a}{b}, B i=\frac{h}{k} \sqrt{\frac{b}{\nu}} \\
& H a=\frac{\sigma B_{0}^{2}}{\rho b}, E c=\frac{U_{\infty}^{2}}{c_{p}\left(T-T_{\infty}\right)}, \operatorname{Pr}=\frac{\rho \nu c_{p}}{k}
\end{aligned}
$$

Substituting equation (5) into equations (1)-(4), we obtain

$$
\begin{aligned}
& f^{\prime \prime \prime}+2 f f^{\prime \prime}-f^{\prime 2}-H a\left(f^{\prime}-1\right)=-1 \\
& \theta^{\prime \prime}+2 \operatorname{Pr} f \theta^{\prime}+\operatorname{PrEc} f^{\prime \prime 2}+\operatorname{PrEcHa}\left(f^{\prime}-1\right)^{2}=0
\end{aligned}
$$

with

$$
\begin{aligned}
& f^{\prime}(0)=\lambda, \quad f(0)=0, \quad \theta^{\prime}(0)=\operatorname{Bi}(\theta(0)-1), \\
& f^{\prime}(\infty)=1, \quad \theta(\infty)=0 .
\end{aligned}
$$

In the above equations, prime denote differentiation with respect to $\eta$, The physical quantities of practical significance in this work are the local skin friction coefficient $C_{f}$ and the local Nusselt number $N u$, which are expressed as

$$
C_{f}=\frac{\tau_{w}}{\rho U_{\infty}^{2}}, \quad N u=\frac{r q_{w}}{k\left(T_{f}-T_{\infty}\right)},
$$

where $C_{f}$ is the skin friction, $\tau$ is the surface shear stress and $q_{w}$ is the heat flux from the plate which are given by

$$
\tau_{w}=\left.\mu \frac{\partial u}{\partial z}\right|_{z=0}, \quad q_{w}=-\left.k \frac{\partial T}{\partial z}\right|_{z=0}
$$

Substituting equation (10) into (9), we obtain

$$
R e_{r}^{1 / 2} C_{f}=f^{\prime \prime}(0), R e_{r}^{-1 / 2} N u=-\theta^{\prime}(0),
$$

where $R e_{r}=U_{\infty} r / \nu$ is the stagnation flow Reynolds number.

\section{METHOD OF SOLUTION}

The set of Equations (6)-(7) together with the boundary conditions (8) are coupled nonlinear which are to be solved numerically using a spectral relaxation method (SRM). This method involves, transforming equations (6)-(7) into a set of linear ordinary differential equations. The entire computation procedure is implemented using a program written in MATLAB computer language.

(4) From the process of numerical computation, the fluid velocity, temperature, the local skin friction coefficient and the local Nusselt number are determined. The SRM algorithm starts with the assumption of having a system of $m$ non-linear ordinary differential equations in $m$ unknowns functions $z_{i}(\eta), \quad i=1,2, \ldots ., m$ where $\eta \in$ $[a, b]$ is the independent variable. To solve the resultant iterative scheme, we then use the Chebyshev pseudospectral method. The details of the spectral methods can be found in (Canuto et al. 1988, Trefethen 2000). Before applying the spectral method, the domain on which the governing equation is defined to the interval $[-1,1]$ on which the spectral method can be implemented. We use the transformation $\eta=(b-a)(\tau+1) / 2$ to map the interval $[a, b]$ to $[-1,1]$.

Now to apply the SRM on (6) together with (7), we set $f^{\prime}(\eta)=g(\eta)$. In view of the SRM, we obtain the following iteration scheme:

$$
\begin{aligned}
& f_{r+1}^{\prime}=g_{r}, \quad f_{r+1}(0)=0, \\
& g_{r+1}^{\prime \prime}+2 f_{r+1} g_{r+1}^{\prime}-\operatorname{Hag}_{r+1}=-1+g_{r}^{2}-H a \\
& g_{r+1}(0)=\lambda, \quad g_{r+1}(\infty)=1 \\
& \frac{1}{P r} \theta_{r+1}^{\prime \prime}+2 f_{r+1} \theta_{r+1}^{\prime}=-E c g_{r+1}^{\prime 2}-\operatorname{EcHa}(g-1)^{2}=0 \\
& \theta_{r+1}(0)=\frac{B i}{1+B i}, \theta_{r+1}(\infty)=0 .
\end{aligned}
$$

We note that the equations now form a system of linear decoupled equations which can be solved iteratively for $r=1,2, \ldots$. , starting from initial guesses/approximations $\left(g_{0}(\eta), \theta(\eta)\right)$.

The initial approximation required to start the iterative process is

$$
\begin{aligned}
& g_{0}(\eta)=\lambda-1+\eta+(1-\lambda) e^{-\eta} \\
& \theta_{0}(\eta)=\frac{B i}{1+B i} e^{-\eta}
\end{aligned}
$$


which are convenient random functions that satisfy the boundary conditions. The iteration is repeated until convergence is achieved. The convergence of the SRM scheme is defined in terms of the infinity norm as

$$
E r=\operatorname{Max}\left(\left\|f_{r+1}-f_{r}\right\| ;\left\|\theta_{r+1}-\theta_{r}\right\|\right)
$$

If the iteration scheme converges, the error $\mathrm{Er}$ is will decrease with an increase in the number of iterations. Accuracy of the scheme was established by increasing the number of collocation points $N$ until the solutions are consistent and further increase does not change the value of the solutions.

\section{Results AND Discussion}

The steady stagnation point flow and heat transfer of an electrically conducting incompressible viscous fluid with the disk surface being convectively heated and radially stretching is numerically solved using the spectral relaxation method (SRM). We have used the bvp4c method to validate the SRM solution. The bvp4c is an in-built MATLAB solver for boundary value problems and is based on fourth order Runge-Kutta schemes. The tolerance level for both methods was set to be $10^{-8}$. The figures were generated using $N=50$ and $\eta_{\infty}=15$. In order to gain physical insight into the flow properties, default numerical values of the parameters encountered in the problem are assigned. Their influences on the velocity and temperature profiles as well as on the skinfriction and the Nusselt number are tabulated and/or displayed graphically.

TABLE I

VALUES OF THE LOCAL NUSSELT NUMBER FOR PARAMETERS BI, EC, $\lambda$ AND HA

\begin{tabular}{lccc}
\hline \hline$B i$ & Ec & $\lambda$ & $-\theta^{\prime}(0)$ \\
\hline 0.0 & & & -0.07207353 \\
0.2 & 0.3 & 0.3 & 0.05773461 \\
0.5 & & & 0.18754275 \\
2.0 & & & 0.44715903 \\
\hline & 0.0 & & 0.25961628 \\
0.3 & 0.2 & 0.3 & 0.18754275 \\
& 0.5 & & 0.07994324 \\
\hline & & 0.0 & 0.02032099 \\
0.3 & 0.3 & 0.2 & 0.11131746 \\
& & 0.5 & 0.22034202 \\
\hline \hline
\end{tabular}

Table 1 displays the effects of the Biot number (Bi), Eckert number (Ec), and the velocity stretching parameter $(\lambda)$ on the Nusselt number $\left(-\theta^{\prime}(0)\right)$. From this table we observe that increasing the Biot number as expected causes the local Nusselt number to increase. Values of the Biot number more than 0.1 imply that the heat convection away from the surface is much faster than the heat inside the body. This gives rise to high temperature gradients at the surface. More heat is transferred from the wall when the values of the Biot number increase thus causing the Nusselt number to increase in our current study. We also observe from this table that as the Eckert number increases, the Nusselt number decreases. Physically, an increase in the Eckert number decreases the temperature gradient between the ambient and the plate. We observe that increasing the values of the Hartman numbers leads to lowering of the values of the Nusselt number. Lastly, we clearly see from the table the Nusselt number is increased when the values of the velocity parameter are increased.

TABLE II

COMPARISON OF SRM SOLUTIONS FOR $f^{\prime \prime}(0)$ AGAINST THOSE OF THE bvp4c FOR $\lambda$ AND HA WHEN PR $=0.71, \mathrm{BI}=0.3, \mathrm{EC}=0.1$

\begin{tabular}{cccc}
\hline \hline & \multicolumn{3}{c}{$f^{\prime \prime}(0)$} \\
\hline$\lambda$ & Ha & SRM & bvp4c \\
\hline 0.0 & & 1.64532167 & 1.64532167 \\
0.2 & 1.0 & 1.38320821 & 1.38320821 \\
0.5 & & 0.92353421 & 1.38320821 \\
\hline & 0.0 & 0.78032335 & 0.78032335 \\
& 1.0 & 0.92353421 & 0.92353421 \\
0.5 & 5.0 & 1.35766817 & 1.35766817 \\
& 10.0 & 1.75767520 & 1.75767520 \\
\hline
\end{tabular}

Table 2 displays the influence of the Hartman number $\mathrm{Ha}$, and velocity parameter $\lambda$. The skin friction is greatly reduced by increasing the velocity parameter. As can be seen from the velocity boundary conditions, $\lambda$ is increased by increasing the stretching parameter $a$. Stretching the surface radially reduces the drag force on the wall surface thus reducing the skin friction. Stretching of the surface may lead to smothering of the surface area thereby reduces the drag force on the wall. We also observe in Table 2 that the Hartman number has significant effect on the skin-friction. As the magnetic strength increases, the dragging effect is clearly seen by the significant increments in the skin friction. The influence of different parameters on the velocity field $f^{\prime}(\eta)$ and temperature profile $\theta(\eta)$ are depicted in Figures 1 to ??. In this table we observe an excellent agreement between the two methods for up to eight decimal places. However, the SRM is converges much faster than the bvp4c both in terms of CPU time and number of iterations. This gives us much confidence in the SRM solutions. 


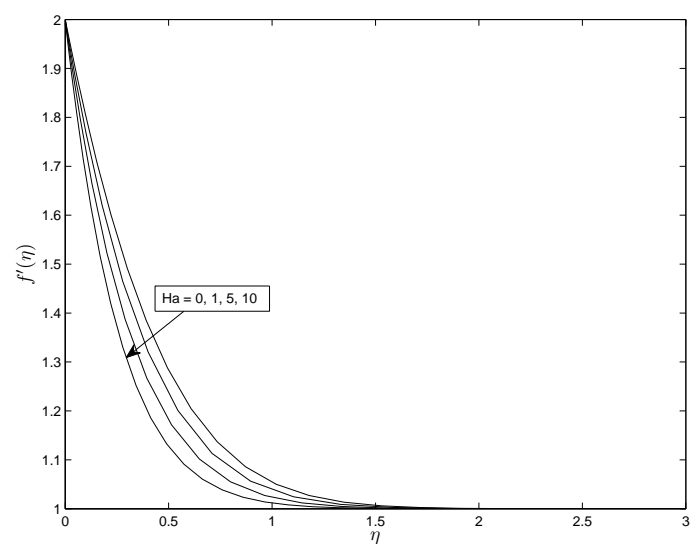

Fig. 1. Influence of $\mathrm{Ha}$ on the velocity profiles when $\lambda=2, \operatorname{Pr}=0.71$, $\mathrm{Bi}=0.3, \mathrm{Ec}=0.2$.

Figure 1 depicts the effects of the Hartmann number $\mathrm{Ha}$ on the velocity distributions. In this figure, we observe that the velocity decreases with $\eta$ as the values of Ha are increased. Thus, the presence of the magnetic field reduces the momentum boundary layer thickness and increases the power needed to stretch the sheet. The presence of a moderate magnetic field can be used to stabilize the flow thereby delaying the transition from laminar to turbulent. Physically, the presence of a transverse magnetic field gives rise to a drag force known as Lorentz force which results in retarding the velocity field. Figure 2 displays the effect of increasing the velocity parameter $\lambda$ on the velocity profiles. Increasing the stretching parameter causes the velocity to increase and reduces the boundary layer. The fluid flow is aided as the radially stretched surface is stretched. This explains why the velocity increases when the stretching parameter increases. The maximum value for the velocity is a unity (one) in dimensionless variables so as the stretching parameter increases, the velocity tend to converge to this value, thus explaining why the boundary layer thickness is reduced.

In Figure 3 we depict the effect of the Biot number on the temperature profiles. The temperature distributions within the fluid flow clearly increase when the Biot number increases. By the mathematical definition of the Biot number increasing its values means that the convective heat transfer coefficient increases thereby enhancing more heat transfer from the surface. This then causes the fluid to heat up thus increasing the fluid temperature distributions. The influence of the Eckert number Ec on the temperature profiles is shown on Figure 4. We observe that the temperature is an increasing function of Ec. This is because increasing the values of the Eckert number generates heat in the fluid due to frictional heating. Thus the effect of increasing Ec is to enhance the temperature at any point.

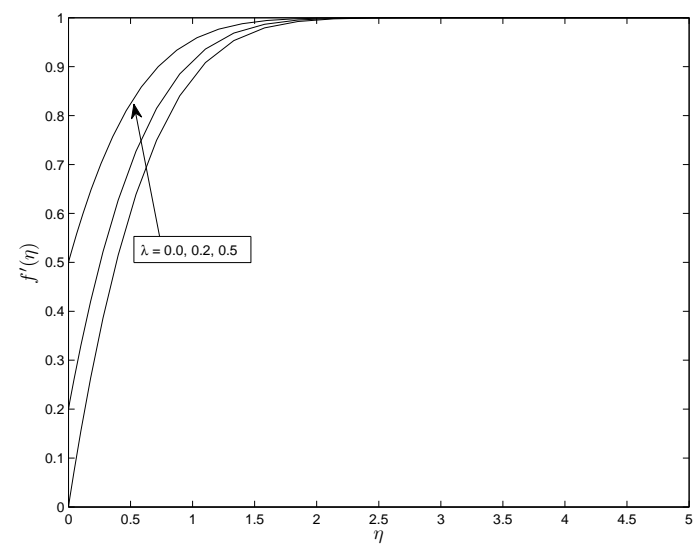

Fig. 2. Variation of the velocity profiles with $\lambda$ when $\mathrm{Pr}=0.71, \mathrm{Ha}=1$, $\mathrm{Bi}=0.3, \mathrm{Ec}=0.3$.

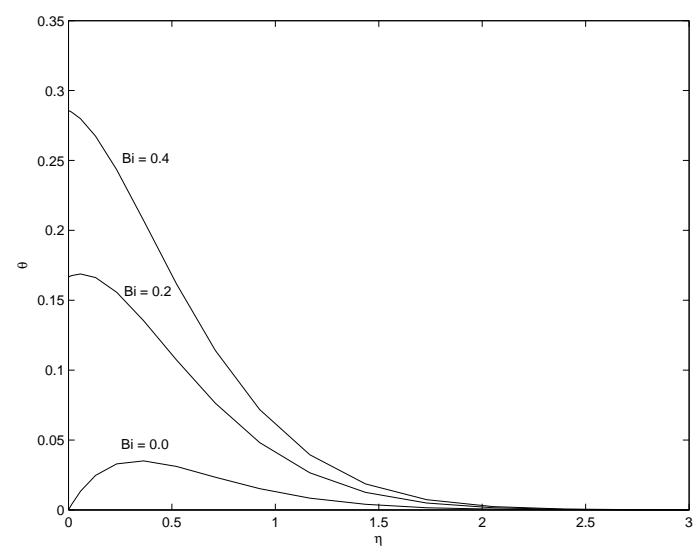

Fig. 3. Influence of Biot number $\mathrm{Bi}$ on the temperature profiles when $\operatorname{Pr}=0.3, \mathrm{Ha}=1, \lambda=0.2, \mathrm{Ec}=0.2$. 


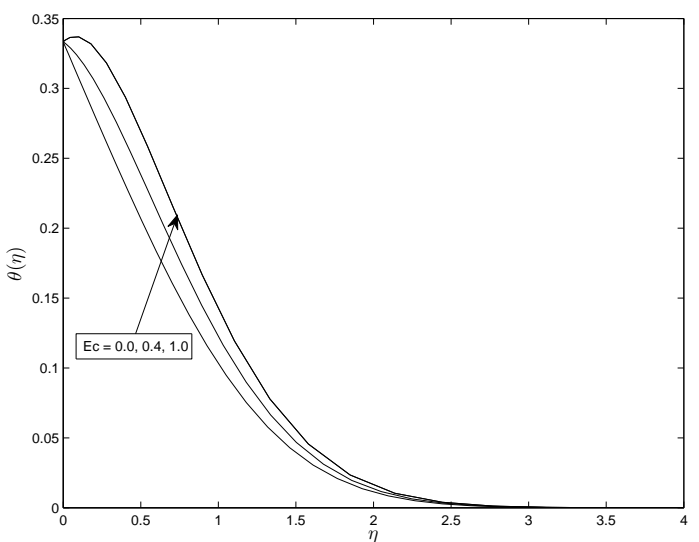

Fig. 4. Influence of Eckert number Ec on the temperature profiles when $\operatorname{Pr}=0.3, \mathrm{Ha}=1, \lambda=0.2, \mathrm{Bi}=0.3$.

Figure 5 displays the effect of increasing the velocity parameter $\lambda$ on the fluid temperature. The temperature is reduced as $\lambda$ increases. Lastly, Figure 6 depicts the effect of the Prandtl number on the temperature. It can clearly observed that the temperature as well as the thermal boundary layer rapidly decrease with increasing values of the Prandtl number. Physically, an increase in the Prandtl number means an increase in fluid velocity which in turn causes a decrease in the temperature.

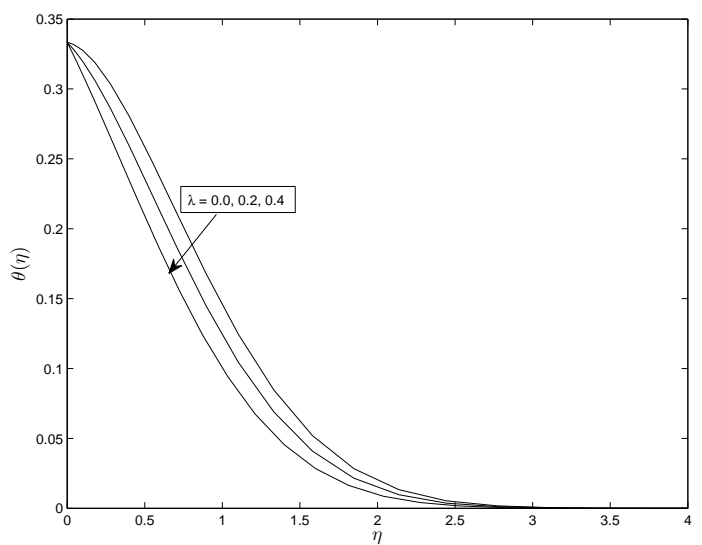

Fig. 5. Influence of velocity parameter on the temperature profiles when $\mathrm{Pr}=0.3, \mathrm{Ha}=1, \mathrm{Bi}=0.3, \mathrm{Ec}=0.2$.

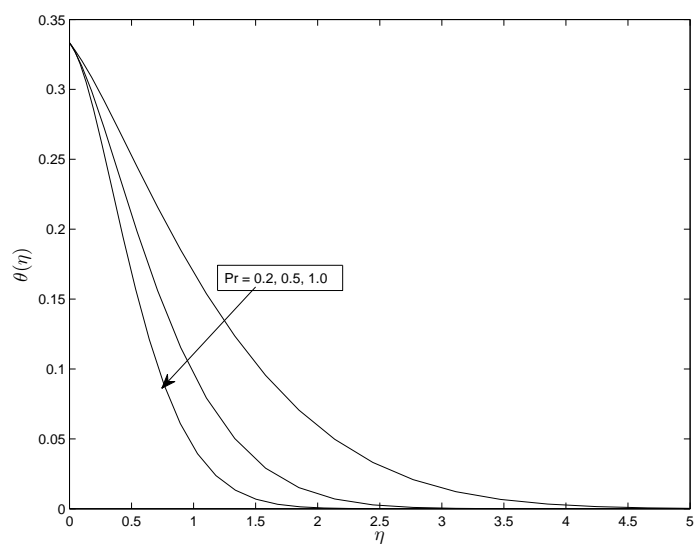

Fig. 6. Variation of the temperature profiles with $\operatorname{Pr}$ when $\mathrm{Bi}=0.3$, $\mathrm{Ha}=1, \lambda=0.2, \mathrm{Ec}=0.2$.

\section{CONCLUSION}

This work is a worthwhile attempt to study the effects of convective heating, viscous dissipation and Joule heating on the stagnation point flow and heat transfer of an electrically conducting fluid towards a radially stretching disk. An effective relaxation spectral algorithm with Chebyshev spectral scheme has been adopted to solve the resulting system of non-linear differential equations subject to the convective boundary conditions. The accuracy of the SRM is validated against the MATLAB inbuilt $b v p 4 c$ routine for solving boundary value problems. The combined effects of the convective heating and the magnetic interaction parameter are studied in detail. The velocity field was found to increase as the Hartmann number and velocity parameter increase. The fluid temperature increases by increasing values of Biot number, Eckert number and the Hartmann number it decreases as the Prandtl number and velocity parameter increase. We also found in this study that the rate of heat transfer at the surface increases as the Biot number (convective parameter) increases.

\section{ACKNOWLEDGMENT}

The authors would like to thank NRF and the University of Venda for financial support.

\section{REFERENCES}

[1] Hiemenz K., Die Grenzschicht in Einem in Dem Gleichformingen Flussigkeitsstrom Eingetauchten Gerade Kreiszlinder, Dingler Polytech Journal. 326 (1911), 321-410.

[2] Motsa S.S., Khan Y., Shateyi S., A New Numerical Solution of Maxwell Fluid over a Shrinking Sheet in the Region of a Stagnation Point, Mathematical Problems in Engineering . Volume 2012, 1-11, doi:10.1155/2012/290615. 
[3] Bhattacharyya, K., Mukhophadhyay, S., Layek, G.C., Slip effects on boundary layer stagnation-point flow and heat transfer towards a shrinking sheet. Int. J. Heat Mass Transf., 54, (2011), 308-313.

[4] Makinde O. D., W. M. Charles W. M., Computational dynamics of hydromagnetic stagnation flow towards a stretching sheet. Applied and Computational Mathematics - Appl. Comput. Math., Vol.9, No.2, (2010), 243-251.

[5] Nadeem, S., Hussain, M., Naz, M., MHD stagnation flow of a micropolar fluid through a porous medium. Meccanica, 45, (2010), 869-880.

[6] Motsa S. S ., Dlamini P.G., M. Khumalo, Solving Hyperchaotic Systems Using the Spectral Relaxation Method, Abstract and Applied Analysis, Volume 2012, 1- 18, doi:10.1155/2012/203461.

[7] Canuto, C., M. Y. Hussaini, A. Quarteroni, and T. A. Zang. (1988) Spectral Methods in Fluid Dynamics, Springer-Verlag, Berlin.

[8] Trefethen, L. N. (2000). Spectral Methods in MATLAB, SIAM, Philadelphia.

[9] Bakar, N. A., Abu Wan Mohd Khairy Adly Wan Zaimi,Rohana Abdul Hamid, Biliana Bidin and 2nuar Ishak., Boundary Layer Flow over a Stretching Sheet with a Convective Boundary Condition and Slip Effect, World Applied Sciences Journal 17 (Special Issue of Applied Math), (2012), 49-53. 